\title{
DIGITALCOMMONS
}

$11-1-2011$

\section{Tests for Correlation on Bivariate Non-Normal Data}

L. Beversdorf

North Carolina State University, louanneb@gmail.com

Ping Sa

University of North Florida, psa@unf.edu

Follow this and additional works at: http://digitalcommons.wayne.edu/jmasm

Part of the Applied Statistics Commons, Social and Behavioral Sciences Commons, and the Statistical Theory Commons

\section{Recommended Citation}

Beversdorf, L. and Sa, Ping (2011) "Tests for Correlation on Bivariate Non-Normal Data," Journal of Modern Applied Statistical Methods: Vol. 10 : Iss. 2 , Article 29.

DOI: $10.22237 /$ jmasm/1320121680

Available at: http://digitalcommons.wayne.edu/jmasm/vol10/iss2/29

This Emerging Scholar is brought to you for free and open access by the Open Access Journals at DigitalCommons@WayneState. It has been accepted for inclusion in Journal of Modern Applied Statistical Methods by an authorized editor of DigitalCommons@WayneState. 


\section{Tests for Correlation on Bivariate Non-Normal Data}

\author{
L. Beversdorf \\ North Carolina State University, \\ Raleigh, NC
}

\author{
Ping $\mathrm{Sa}$ \\ University of North Florida, \\ Jacksonville, FL
}

Two statistics are considered to test the population correlation for non-normally distributed bivariate data. A simulation study shows that both statistics control type I error rates well for left-tailed tests and have reasonable power performance.

Key words: Saddlepoint approximation, Fisher's transformation, tests for correlation, bivariate nonnormal distribution.

\section{Introduction}

Bivariate data are data in which two variables are measured on an individual. If the variables are quantitative, a researcher may be interested in describing the relationship between them. One measure used to describe the strength of linear relation between two quantitative variables is the linear correlation coefficient, denoted by $\rho$.

The true relationship between two variables of interest is always unknown. Different estimators have been proposed for $\rho$ and two of them are used frequently: (1) the Spearman Rank Order Correlation, which is used for ordinal data, and (2) the Pearson Product Moment Correlation, which is applied to interval and ratio data. The maximum likelihood estimator of $\rho$ is the Pearson product-moment

Ping $\mathrm{Sa}$ is a Professor of the Department of Mathematics and Statistics. She received her Ph.D. in Statistics from the University of South Carolina in 1990. She has published more than 20 papers. Her recent scholarly activities have involved research in multiple comparisons, quality control and statistical inference for nonnormal data. Email: psa@unf.edu. Ms. Beversdorf received her Master's degree in Statistics from the University of North Florida in 2008. She is currently a Ph. D. candidate at North Carolina State University. Email: louanneb@gmail.com. correlation coefficient. When the data is not bivariate normal and the sample size exceeds 10 , the nonparametric Spearman rank correlation is useful. Little work has been done for cases when the distribution of the data is unknown and the sample size is relatively small.

The most popular $\rho$ estimator is the Pearson Product Moment Correlation Coefficient, $r$, which is a biased point estimator for $\rho$, however, the bias is small when $n$ (sample size) is large. Given two variables $Y_{1}$ and $Y_{2}$, the statistic is:

$$
r=\frac{\sum_{i=1}^{n}\left(Y_{i 1}-\bar{Y}_{1}\right)\left(Y_{i 2}-\bar{Y}_{2}\right)}{\left[\sum_{i=1}^{n}\left(Y_{i 1}-\bar{Y}_{1}\right)^{2}\left(Y_{i 2}-\bar{Y}_{2}\right)^{2}\right]^{1 / 2}},
$$

where $\left(Y_{i 1}, Y_{i 2}\right)$ is the $\mathrm{i}^{\text {th }}$ observation of the bivariate data $\left(\mathrm{Y}_{11}, \mathrm{Y}_{12}\right), \ldots,\left(\mathrm{Y}_{\mathrm{n} 1}, \mathrm{Y}_{\mathrm{n} 2}\right), \bar{Y}_{1}$ is the sample mean of $Y_{1}$ and $\bar{Y}_{2}$ is the sample mean of $\mathrm{Y}_{2}$.

Researchers have done intensive work on the distribution of $r$ when the population is bivariate normal (Fisher, 1915; Stuart \& Ord, 1994). It has been found that, when $n=2$, the distribution of $r$ can be regarded as an extreme case of a U-shaped distribution, for $n=3$ the density is still U-shaped, but if $n=4$ the distribution is uniform when $\rho=0$ and $\mathrm{J}$-shaped otherwise. For $n>4$ the density function is unimodal and has increased skew as $|\rho|$ increases, this follows from the fact that the 


\section{TESTS FOR CORRELATION ON BIVARIATE NON-NORMAL DATA}

mode moves with $\rho$ and $r$. For any $\rho$, the distribution of $r$ slowly tends to normality as $n$ $\rightarrow \infty$ (Stuart \& Ord, 1994).

When the population is bivariate normal and has equal variance, a test statistic

$$
t_{r}^{*}=\frac{r \sqrt{n-2}}{\sqrt{1-r^{2}}}
$$

can be derived to test $\mathrm{H}_{0}: \rho=0$. Under $\mathrm{H}_{0}, t_{r}^{*}$ follows the Student's $t$-distribution with $(n-2)$ degrees of freedom, denoted $t_{(n-2)}$. Disadvantages of this test include the need for a relatively large sample or bivariate normal data and the ability to test only for $\rho=0$.

When the population is not bivariate normal and the sample size exceeds 10 , a nonparametric statistic, the Spearman Rank Correlation Coefficient (Spearman), is typically used to measure the association between two variables when no transformation for the data can be found to approximate a bivariate normal distribution. Spearman, denoted by $r_{s}$, is then defined as the ordinary Pearson product-moment correlation coefficient based on data ranking:

$$
r_{s}=\frac{\sum\left(R_{i 1}-\bar{R}_{1}\right)\left(R_{i 2}-\bar{R}_{2}\right)}{\left.\sum\left(R_{i 1}-\bar{R}_{1}\right)^{2}\left(R_{i 2}-\bar{R}_{2}\right)^{2}\right]^{1 / 2}},
$$

where $\left(\mathrm{R}_{\mathrm{i} 1}, \mathrm{R}_{\mathrm{i} 2}\right)$ are the ranks of $\left(Y_{i 1}, Y_{i 2}\right)$ respectively; and $\bar{R}_{1}$ is the mean of the ranks of $\mathrm{R}_{\mathrm{i} 1}, \mathrm{i}=1,2, \ldots \mathrm{n}$, and $\bar{R}_{2}$ is the mean of the ranks of $R_{i 2}, i=1,2, \ldots n$.

Spearman can also be used to test the association between the two variables with the null hypothesis, $\mathrm{H}_{0}$, stating: there is no association between $Y_{1}$ and $Y_{2}$. When sample size $n$, exceeds 10 , the test statistic:

$$
t_{r s}^{*}=\frac{r_{s} \sqrt{n-2}}{\sqrt{1-r_{s}^{2}}}
$$

can be used. $t_{r s}^{*}$ is approximately a $t$-distribution with $n-2$ degrees of freedom under $\mathrm{H}_{0}$. This is a nonparametric test and thus may result in lower power performance. Again this test can only be used for testing whether an association exists. The purpose of this study is to test $\mathrm{H}_{0}: \rho=\rho_{0}$, where $\rho_{0}$ can be values other than zero, for bivariate non-normal data. Fisher's Ztransformation and a saddlepoint transformation are investigated and tested.

\section{Methodology}

Two statistics for testing the correlation coefficient of bivariate non-normal populations are investigated: (1) Fisher's z-transformation, denoted $r_{F}$, and (2) the saddlepoint approximation, denoted $r_{L}$. These methods are used on bivariate non-normal data sets with small sample sizes. The goal is to determine if either of the two methods is appropriate for hypothesis testing about the population correlation coefficient, specifically for bivariate non-normal data sets with a small sample size.

Fisher's Z-Transformation

The sampling distribution of $r$ is complicated when $\rho \neq 0$ even when the population is bivariate normal. Fisher (1921) derived an approximation procedure based on a transformation of $r, z^{\prime}=\frac{1}{2} \log \frac{1+r}{1-r}$ and it tends to normality much faster than $r$. After standardizing, the statistic for Fisher's classical transformation is given by:

$$
r_{F}=\left(\frac{1}{2} \log \frac{1+r}{1-r}-\frac{1}{2} \log \frac{1+\rho}{1-\rho}-\frac{\rho}{2(n-1)}\right) \sqrt{n-3} .
$$

Saddlepoint Approximation

Saddlepoint approximations were introduced by Daniels (1954). However, computations of these approximations only recently became feasible with the availability of inexpensive computing power. In practice, statistical inference often involves test statistics with normal distributions, which are valid as sample sizes increase. For small sample size problems, these distributions tend to provide inaccurate results. Saddlepoint methods offer approximations that are accurate to a higher order than first-order approximations and their 


\section{BEVERSDORF \& SA}

accuracy holds for extremely small sample sizes (Huzurbazar, 1999). Saddlepoint approximations also provide good estimates to very small tail probabilities or to the density in the tails of the distributions.

Jensen (1995) transforms the Pearson correlation coefficient using Laplace transformations to derive a function of $r$ that can be normalized and he claims that $r_{L}$ is normally distributed to a high accuracy. Assuming a bivariate normal data set with correlation $\rho$, the saddlepoint approximation, denoted $r_{L}$, provided by Jensen (1995) is:

$$
r_{L}=v+\frac{1}{v} \log \frac{u}{v}
$$

where

$$
\begin{gathered}
v=\operatorname{sgn}(r-\rho)\left\{2(n-4) \log \left(\frac{1-\rho r}{\sqrt{1-\rho^{2}} \sqrt{1-r^{2}}}\right)\right\}^{\frac{1}{2}}, \\
u=\sqrt{n-4}\left(\frac{1-\rho r}{1-\rho^{2}}\right)^{\frac{3}{2}} \frac{r-\rho}{1-r^{2}}
\end{gathered}
$$

and $\operatorname{sgn}($.$) is the sign of (r-\rho)$.

\section{Proposed Test}

A new test is required to investigate the hypothesis $H_{0}: \rho=\rho_{0}$ versus three possible alternative hypotheses, $H_{a}: \rho \neq \rho_{0}, \quad H_{a}: \rho>\rho_{0}$ and $H_{a}: \rho<\rho_{0}$, when a data set is bivariate nonnormal and sample size is relatively small to moderate. Although both the Fisher and saddlepoint transformations are derived for bivariate normal data, little work has been done to investigate if they can also be used for nonnormal bivariate data; thus, the two approximations, $r_{F}$ in (3) and $r_{L}$ in (4), are used as the test statistics for the hypothesis $\mathrm{H}_{0}: \rho=\rho_{0}$. Note that $\rho_{0}$ should be used in both equations whenever $\rho$ is present. The decision rule to reject the null hypothesis for the twotailed, upper-tailed and lower-tailed tests is $\left|r_{F}\right|$ $>\mathrm{z}_{\alpha / 2}$ or $\left|r_{L}\right|>\mathrm{z}_{\alpha / 2}, r_{F}, r_{L}>\mathrm{z}_{\alpha}$, and $r_{F}, r_{L}<-\mathrm{z}_{\alpha,}$, respectively.

Simulation Study: Generating Bivariate NonNormal Data

Fleishman (1978) derived a method for generating univariate non-normal random variables. Fleishman's method is based on the variable $\mathrm{Y}$ defined as

$$
Y=a+b Z+c Z^{2}+d Z^{3}
$$

where $\mathrm{Z}$ is a standard normal random variable, and $a, b, c$ and $d$ are constants chosen in such a way that $\mathrm{Y}$ has the desired coefficients of skewness and kurtosis, $\gamma_{1}$ and $\gamma_{2}$, respectively.

Fleishman showed that $a=-c$ and the constants $b, c$ and $d$ are determined by simultaneously solving the following three equations:

$$
\begin{aligned}
& b^{2}+6 b d+2 c^{2}+15 d^{2}-1=0 \\
& 2 c\left(b^{2}+24 b d+105 d^{2}+2\right)-\gamma_{1}=0 \\
& 24\left\{\begin{array}{l}
b d+c^{2}\left(1+b^{2}+28 b d\right) \\
+d^{2}\left(12+48 b d+141 c^{2}+225 d^{2}\right)
\end{array}\right\}-\gamma_{2}=0
\end{aligned}
$$

Using these equations, a non-normal random variable $\mathrm{Y}$ can be obtained by generating a standard normal variable $\mathrm{Z}$ and using the equation (5).

Vale and Maurelli (1983) proposed generating multivariate non-normal random variables with a specified correlation structure based on Fleishman's method. For bivariate nonnormal random data, $\left(\mathrm{Y}_{1}, \mathrm{Y}_{2}\right)$ with desired coefficients of skewness and kurtosis, $\left(\gamma_{11}\right.$ and $\gamma$ $\left.{ }_{21}\right)$ for $Y_{1}$ and $\left(\gamma_{12}\right.$ and $\left.\gamma_{22}\right)$ for $Y_{2}$, solutions to the system of equations (6) given in Fleishman's method must be found. Let $Z_{1}, Z_{2}$ be two standard normal correlated variables. $\mathrm{Y}_{1}$ and $\mathrm{Y}_{2}$ can be calculated with the following equations:

$$
\begin{aligned}
& Y_{1}=a_{1}+b_{1} Z_{1}+c_{1} Z_{1}^{2}+d_{1} Z_{1}^{3}, \\
& Y_{2}=a_{2}+b_{2} Z_{2}+c_{2} Z_{2}^{2}+d_{2} Z_{2}^{3}
\end{aligned}
$$




\section{TESTS FOR CORRELATION ON BIVARIATE NON-NORMAL DATA}

Vale and Maurelli (1983) found that the correlation coefficient between $Y_{1}$ and $Y_{2}$ is:

$$
\begin{aligned}
& \rho_{Y_{1}, Y_{2}}= \\
& \quad \rho_{Z_{1}, Z_{2}}\left(b_{1} b_{2}+3 b_{1} d_{2}+3 b_{2} d_{1}+9 d_{1} d_{2}\right) \\
& +\rho_{Z_{1}, Z_{2}}^{2} 2 c_{1} c_{2}+\rho_{Z_{1}, Z_{2}}^{3} 6 d_{1} d_{2}
\end{aligned}
$$

For a desired correlation, $\rho_{y_{1}, y_{2}}$, the intermediate correlation, $\rho_{Z_{1}, Z_{2}}$, can be determined by solving the above cubic equation. The bivariate nonnormal random variate $\left(\mathrm{Y}_{1}, \mathrm{Y}_{2}\right)$ can then be obtained by first generating a set of bivariate standard normal random variate with correlation $\rho_{Z_{1}, Z_{2}}$, and then using equation (7).

\section{Simulation Description}

Different values of skewness and kurtosis were chosen for the simulation study in order to reflect different population distributions. Four values of skewness, $-3,-1$, 1,3 and three values of kurtosis, 3, 7, 25 were used, resulting in 78 possible pairs of populations. A relatively small sample size of 10 and a moderate sample size of 20 were used in the study and the test statistics $r_{L}$ and $r_{F}$ were investigated for type I error rates with left-tailed, right-tailed and two-tailed tests with the nominal levels of 0.01 and 0.05 for each sample.

Comparisons in the simulation study use $r_{L}$ and $r_{F}$ against three critical values, $\mathrm{z}_{\alpha}, \mathrm{t}_{(\mathrm{n}-2, \alpha)}$, and $\left(\mathrm{z}_{\alpha}+\mathrm{t}_{(\mathrm{n}-2, \alpha)}\right) / 2$, to draw conclusions. Four $\rho_{0}$ values $0,0.5,0.7$ and 0.9 were evaluated as the hypothesized values for $\mathrm{H}_{0}: \rho=\rho_{0}$. When $\rho_{0}=0$, the $t_{r}^{*}$ in (1) and $t_{r s}^{*}$ in (2) are also included in the study for comparison purposes. The simulation study has two parts: the type I error rate comparisons and the power study. The steps of the simulation are:

Data Generation: Steps (1) - (5)

1) Input the five population parameters: skewness and kurtosis for each of the two populations and the desired population correlation;
2) Solve the system of equations (6) to calculate coefficients $a, b, c$ and $d$ for the two populations;

3) Solve $\rho_{z_{1} z_{2}}$ by equation (8);

4) Generate $n$ bivariate standard normal variables $\left(Z_{1}, Z_{2}\right)$ with correlation $\rho_{z_{1} z_{2}}$;

5) Apply the transformation in (7) to obtain the non-normal sample data $Y_{1}$ and $Y_{2}$;

Evaluation: Steps (6) - (8)

6) Evaluate $r_{L}$ and $r_{F}$ and compare to critical values $\mathrm{z}_{\alpha}, \mathrm{t}_{(\mathrm{n}-2, \alpha)}$, and $\left(\mathrm{z}_{\alpha}+\mathrm{t}_{(\mathrm{n}-2, \alpha)}\right) / 2$; if $\rho_{0}=0$, $t_{r}^{*}$ and $t_{r s}^{*}$ are evaluated and compared to $t_{(n-}$ 2) critical value;

7) Repeat steps (4) - (6) 99,999 times;

8) Calculate type I error rate for each method by finding the proportion of rejection in the 100,000 samples.

In the power study, an extra parameter $\rho_{\mathrm{a}}$ (which is different from $\rho_{0}$ ) is input in step (1) and used to generate the data as the true population correlation, however, all test statistics in step (6) are evaluated under $\rho_{0}$. All other steps in the power study are identical to the type I error rate study. All the simulations were run with Fortran 77 for Windows on a Toshiba Satellite-A105 Laptop Computer.

\section{Results}

\section{Type I Error Rate Comparison}

Tables 1-4 provide comparisons of type I error rates with sample size $n=10$. The set of population parameters for skewness and kurtosis are in the first column with the first population's parameters in the first row and the second in the second row. Comparisons were made between the tests for saddlepoint and Fisher's transformation, given in the table as the two adjacent numbers within a given correlation column, $r_{L}$ and $r_{F}$, respectively. Three critical 


\section{BEVERSDORF \& SA}

points $t_{n-2, \alpha}, \frac{z_{\alpha}+t_{n-2, \alpha}}{2}$ and $z_{\alpha}$ were used for the two proposed methods. The results are the first, second and third numbers in the respective column. Pearson and Spearman are evaluated with a critical value $t_{n-2, \alpha}$ for $\rho=0$ only, and the type I error rates are reported in the first column with Pearson first and Spearman underneath.

Due to similar results in the study, only 12 pairs of populations and the small sample size $\mathrm{n}=10$ are reported in the tables. Also, although all the tests are done with levels of significance 0.05 and 0.01 , both levels are reported here only for the left-tailed tests. (For complete simulation results, please contact the first author.)

\section{Left-Tailed Type I Error Rates}

Left-tailed type I error rates are given in Tables 1 and 2. Table 1 uses a significance level of 0.05 and Table 2 uses a significance level of 0.01 . It can be observed that only slight differences in type I error rates are present between the results for the saddlepoint and Fisher's transformations. This same result was observed throughout the simulation study.

Results using the $t$ critical value achieve very good type I error rates for all of the distributions. The $z$ critical value results in a few slightly inflated type I error rates and only by the saddlepoint approximation. The worst case found in the study, produced by the saddlepoint approximation, is for the pair populations with the same (skewness, kurtosis) $=(3,25)$ under $\rho$ $=0.9$ using $z_{\alpha}$ as the critical point. The type I error rate for this case is 0.0688 . However, after the critical point was changed to $\frac{z_{\alpha}+t_{n-1, \alpha}}{2}$, the type I error rate decreased to 0.0564 and it further decreased to 0.0458 when $t_{n-2, \alpha}$ is used. The Fisher's transformation, by contrast, controls the type I error rates properly for nearly all cases considered.

For the important case when $\rho=0$, results show that both the $r_{L}$ and $r_{F}$ statistics control type I error rates using any of the three critical values at the 0.05 significance level. When the significance level is lowered to 0.01 , some of the type I error rates using the $z$ critical value are slightly inflated but within acceptable range. Surprisingly, Pearson controls the type I error rates better than the Spearman method. It performed very well for the 0.05 significance level; however, those involving a population with larger kurtosis are slightly inflated when the significance level is lowered to 0.01 . Spearman has some slightly inflated type I error rates at both significance levels. Overall, it is fair to say that essentially all cases studied produced controlled type I error rates for the left-tailed test.

Right-Tailed \& Two-Tailed Type I Error Rates Right-tailed type I error rates are shown in Tables 3 with significance level of 0.05 . (Although the 0.01 level of significance is also studied, the table is omitted due to the similar results.) With the right-tailed test, most type I error rates are inflated, the only values that stand out are for tests where the $t$ critical values were used and both the skewness and kurtosis were relatively small. A great result is found for the $t$ critical values when $\rho=0$, type I error rates are controlled for both the $r_{L}$ and $r_{F}$. As opposed to the left-tailed test, the Spearman $t$-test works better than the Pearson; however, results are still not as good as the corresponding results by $r_{L}$ and $r_{F}$.

Overall, both Saddlepoint and Fisher's statistics are better candidates for testing $\mathrm{H}_{\mathrm{a}}: \rho>0$. The $t$ critical value produces more stable results then the $z$ critical value, although the two statistics can also be used for other $\rho_{0}$ values if the populations have small kurtosis with $t$ critical points, in general the two statistics are not recommended for a right-tailed test.

Two-tailed type I error rates are shown in Table 4. As expected, the results of the twotailed tests are more controlled than that of the right-tailed test. However, because the methods essentially failed for the right-tailed tests, they are not recommended to be used to perform a two-tailed test.

Power Results

Table 5 summarizes the results of the power study for left-tailed tests with $H_{0}: \rho=0.7$ versus various $\rho_{\mathrm{a}}$ values such that 


\section{TESTS FOR CORRELATION ON BIVARIATE NON-NORMAL DATA}

$\rho_{\mathrm{a}}<0.7$. Five different $\rho_{\mathrm{a}}$ values and two levels of significance were investigated, but only three $\rho_{\mathrm{a}}$ and $\alpha=0.05$ results are reported here. Power results for both methods show reasonable rate of convergence to probability 1 . As expected, the $z$ critical values have higher power than the other two tests. (For complete simulation results, please contact the first author.)

\section{Conclusion}

This study proposed and examined two statistics, the saddlepoint transformation, $r_{L}$, and Fisher's transformation, $r_{F}$, for testing a correlation which may or may not be zero for any bivariate non-normal population. The simulation study indicates that the two statistics perform similarly. They both have very good robust performance for all the distributions studied when testing a left-tailed test; they maintain the type I error rates close to the nominal level and show reasonably good power.

The two statistics are not recommended for testing a right-tailed test or a two-tailed test unless the practitioner knows for certain that the populations have both small skewness and kurtosis. In these cases, the two test statistics with a $t$ critical point can properly control the type I error rates.

The two statistics can also be used for testing $\mathrm{H}_{0}: \rho=0$ versus any of the three possible alternative hypotheses. They control type I error rates better than the existing Pearson and Spearman $t$-tests. Because the two statistics are derived based on bivariate normal population, a sample size of at least 10 is recommended.

\section{References}

Daniels, H. E. (1954). Saddlepoint approximations in statistics. Annals of Mathematical Statistics, 25, 631-650.

Fisher, R. A. (1915). Frequency distribution of the values of the correlation coefficient in samples from an indefinitely large population. Biometrika, 10(4), 507-521.

Fisher, R. A. (1921). On the probable error of a coefficient of correlation deduced from a small sample. Metron, 1(4), 1.

Fleishman, A. (1978). A method for simulating non-normal distribution.

Psychometrika, 43, 521-532.

Huzurbazar, S. (1999). Practical saddlepoint approximation. The American Statistician, 53(3), 225-232.

Jensen, J. (1995). Saddlepoint approximation. New York: Oxford University Press, Inc.

Stuart, A., \& Ord, J. K. (1994). Kendall's advanced theory of Statistics, Vol. 1, $6^{\text {th }} E d$. New York: Halsted Press.

Vale, C., \& Maurelli, V. (1983). Simulating multivariate non-normal distributions. Psychometrika, 48, 465-471. 
BEVERSDORF \& SA

Table 1: Type I Error Rates for Left-Tailed Test, 0.05 Level of Significance

\begin{tabular}{|c|c|c|c|c|c|c|c|c|c|c|}
\hline \multirow[b]{2}{*}{ Skewness } & \multirow[b]{2}{*}{ Kurtosis } & \multicolumn{3}{|c|}{$\mathrm{RHO}=0$} & \multicolumn{2}{|c|}{$\mathrm{RHO}=0.5$} & \multicolumn{2}{|c|}{$\mathrm{RHO}=0.7$} & \multicolumn{2}{|c|}{$\mathrm{RHO}=0.9$} \\
\hline & & $\begin{array}{c}\text { Pearson } \\
\text { Spearman }\end{array}$ & $r_{L}$ & $r_{F}$ & $\mathrm{r}_{\mathrm{L}}$ & $\mathrm{r}_{\mathrm{F}}$ & $\mathrm{r}_{\mathrm{L}}$ & $r_{F}$ & $r_{L}$ & $r_{F}$ \\
\hline 3 & 25 & 0.0416 & 0.0284 & 0.0281 & 0.021 & 0.0179 & 0.0323 & 0.0263 & 0.0458 & 0.0356 \\
\hline \multirow[t]{2}{*}{3} & 25 & 0.0522 & 0.0348 & 0.0339 & 0.029 & 0.0248 & 0.0421 & 0.0344 & 0.0564 & 0.0447 \\
\hline & & & 0.0424 & 0.0408 & 0.0395 & 0.0334 & 0.0537 & 0.0444 & 0.0688 & 0.055 \\
\hline-3 & 25 & 0.0428 & 0.0292 & 0.0289 & 0.0218 & 0.0188 & 0.0325 & 0.0261 & 0.0445 & 0.0342 \\
\hline \multirow[t]{2}{*}{-3} & 25 & 0.0538 & 0.0357 & 0.0348 & 0.0298 & 0.0255 & 0.0429 & 0.0348 & 0.0553 & 0.0433 \\
\hline & & & 0.0436 & 0.042 & 0.0397 & 0.0337 & 0.0549 & 0.0451 & 0.0674 & 0.054 \\
\hline-1 & 7 & 0.0475 & 0.0302 & 0.0298 & 0.0285 & 0.0256 & 0.0289 & 0.0241 & 0.0311 & 0.0235 \\
\hline \multirow[t]{2}{*}{-1} & 7 & 0.0516 & 0.0381 & 0.037 & 0.0368 & 0.0324 & 0.0369 & 0.0307 & 0.0395 & 0.0302 \\
\hline & & & 0.0483 & 0.0463 & 0.0462 & 0.0409 & 0.0469 & 0.0387 & 0.0502 & 0.0383 \\
\hline 1 & 7 & 0.0483 & 0.0316 & 0.0312 & 0.0283 & 0.0252 & 0.0292 & 0.0245 & 0.0316 & 0.0235 \\
\hline \multirow[t]{2}{*}{1} & 7 & 0.0521 & 0.0396 & 0.0385 & 0.036 & 0.0318 & 0.0374 & 0.0312 & 0.0403 & 0.0307 \\
\hline & & & 0.0491 & 0.0473 & 0.0453 & 0.0399 & 0.0475 & 0.0392 & 0.0515 & 0.0392 \\
\hline 1 & 3 & 0.0463 & 0.0286 & 0.0281 & 0.0309 & 0.0277 & 0.0321 & 0.0268 & 0.0354 & 0.0276 \\
\hline \multirow[t]{2}{*}{1} & 3 & 0.0514 & 0.0374 & 0.0362 & 0.0398 & 0.0351 & 0.0411 & 0.034 & 0.0448 & 0.0345 \\
\hline & & & 0.0473 & 0.0454 & 0.0501 & 0.0444 & 0.0527 & 0.0434 & 0.0555 & 0.0434 \\
\hline-1 & 3 & 0.0461 & 0.0286 & 0.028 & 0.0313 & 0.0278 & 0.0326 & 0.0271 & 0.0349 & 0.0264 \\
\hline & 3 & 0.0517 & 0.0371 & 0.0358 & 0.0399 & 0.0355 & 0.0411 & 0.0346 & 0.0438 & 0.0338 \\
\hline & & & 0.047 & 0.0449 & 0.0505 & 0.0444 & 0.0517 & 0.0433 & 0.055 & 0.0426 \\
\hline-3 & 25 & 0.0441 & 0.0278 & 0.0272 & 0.0228 & 0.0198 & 0.0189 & 0.0153 & 0.002 & 0.0013 \\
\hline & 3 & 0.0519 & 0.0357 & 0.0346 & 0.0304 & 0.0264 & 0.025 & 0.0203 & 0.0029 & 0.0019 \\
\hline & & & 0.045 & 0.0431 & 0.0392 & 0.0343 & 0.0328 & 0.0266 & 0.0043 & 0.0028 \\
\hline 3 & 25 & 0.0446 & 0.0284 & 0.0279 & 0.0226 & 0.0201 & 0.0199 & 0.0163 & 0.0019 & 0.0012 \\
\hline \multirow[t]{2}{*}{1} & 3 & 0.0512 & 0.0359 & 0.0349 & 0.0293 & 0.0259 & 0.026 & 0.0212 & 0.0027 & 0.0018 \\
\hline & & & 0.0455 & 0.0436 & 0.0382 & 0.0329 & 0.0341 & 0.0274 & 0.0043 & 0.0026 \\
\hline 3 & 25 & 0.0464 & 0.0307 & 0.0302 & 0.0221 & 0.0194 & 0.0217 & 0.0175 & 0.0112 & 0.0079 \\
\hline \multirow[t]{2}{*}{1} & 7 & 0.0521 & 0.0383 & 0.0371 & 0.029 & 0.0253 & 0.0288 & 0.023 & 0.0151 & 0.0109 \\
\hline & & & 0.0472 & 0.0454 & 0.0375 & 0.0325 & 0.0377 & 0.0306 & 0.0207 & 0.0146 \\
\hline-3 & 25 & 0.0474 & 0.0317 & 0.0312 & 0.0213 & 0.0187 & 0.0219 & 0.0178 & 0.0115 & 0.0081 \\
\hline \multirow[t]{2}{*}{-1} & 7 & 0.0527 & 0.0389 & 0.0379 & 0.028 & 0.0244 & 0.0288 & 0.0235 & 0.0158 & 0.011 \\
\hline & & & 0.0482 & 0.0464 & 0.0366 & 0.0318 & 0.0375 & 0.0303 & 0.0212 & 0.0151 \\
\hline-1 & 3 & 0.0482 & 0.0311 & 0.0304 & 0.0293 & 0.0263 & 0.0296 & 0.0244 & 0.0254 & 0.019 \\
\hline & 7 & 0.0521 & 0.0389 & 0.0379 & 0.0374 & 0.0331 & 0.0373 & 0.0313 & 0.0333 & 0.0247 \\
\hline & & & 0.0489 & 0.0473 & 0.0471 & 0.0415 & 0.0469 & 0.0392 & 0.0422 & 0.0321 \\
\hline 1 & 3 & 0.0473 & 0.0301 & 0.0294 & 0.0299 & 0.0267 & 0.0298 & 0.025 & 0.0258 & 0.0194 \\
\hline \multirow[t]{2}{*}{1} & 7 & 0.0522 & 0.038 & 0.0366 & 0.038 & 0.0338 & 0.0379 & 0.0315 & 0.0331 & 0.0251 \\
\hline & & & 0.0481 & 0.0461 & 0.0482 & 0.0424 & 0.0475 & 0.0396 & 0.0419 & 0.0321 \\
\hline
\end{tabular}




\section{TESTS FOR CORRELATION ON BIVARIATE NON-NORMAL DATA}

Table 2: Type I Error Rates for Left-Tailed Test, 0.01 Level of Significance

\begin{tabular}{|c|c|c|c|c|c|c|c|c|c|c|}
\hline \multirow[b]{2}{*}{ Skewness } & \multirow[b]{2}{*}{ Kurtosis } & \multicolumn{3}{|c|}{$\mathrm{RHO}=0$} & \multicolumn{2}{|c|}{$\mathrm{RHO}=0.5$} & \multicolumn{2}{|c|}{$\mathrm{RHO}=0.7$} & \multicolumn{2}{|c|}{$\mathrm{RHO}=0.9$} \\
\hline & & $\begin{array}{c}\text { Pearson } \\
\text { Spearman }\end{array}$ & $r_{L}$ & $\mathrm{r}_{\mathrm{F}}$ & $r_{L}$ & $\mathrm{r}_{\mathrm{F}}$ & $r_{L}$ & $r_{F}$ & $r_{L}$ & $r_{F}$ \\
\hline 3 & 25 & 0.0113 & 0.0039 & 0.0049 & 0.0008 & 0.0011 & 0.0009 & 0.001 & 0.0021 & 0.0019 \\
\hline \multirow[t]{2}{*}{3} & 25 & 0.0118 & 0.0069 & 0.0079 & 0.0021 & 0.0023 & 0.0028 & 0.0027 & 0.0063 & 0.0049 \\
\hline & & & 0.0115 & 0.0123 & 0.0049 & 0.0047 & 0.0077 & 0.0067 & 0.0142 & 0.011 \\
\hline-3 & 25 & 0.0114 & 0.0033 & 0.0043 & 0.0006 & 0.0008 & 0.001 & 0.001 & 0.0023 & 0.002 \\
\hline & 25 & 0.0119 & 0.0063 & 0.0073 & 0.0019 & 0.002 & 0.0028 & 0.0026 & 0.0061 & 0.0047 \\
\hline & & & 0.0117 & 0.0124 & 0.0051 & 0.0049 & 0.008 & 0.0068 & 0.0149 & 0.0113 \\
\hline-1 & 7 & 0.0105 & 0.0023 & & 0.0016 & 0.002 & 0.0016 & 0.0017 & 0.0017 & 0.0016 \\
\hline & 7 & 0.0117 & 0.0052 & 0.0063 & 0.0037 & 0.004 & 0.0036 & 0.0035 & 0.004 & 0.0032 \\
\hline & & & 0.0108 & 0.0118 & 0.009 & 0.0088 & 0.0086 & 0.0074 & 0.0097 & 0.0073 \\
\hline 1 & 7 & 0.0105 & 0.0022 & 0.0032 & 0.0015 & 0.0019 & 0.0015 & 0.0017 & 0.0017 & 0.0015 \\
\hline & 7 & 0.0112 & 0.0051 & 0.0059 & 0.004 & 0.0042 & 0.0039 & 0.0038 & 0.0041 & 0.0033 \\
\hline & & & 0.0108 & 0.0118 & 0.0086 & 0.0084 & 0.0088 & 0.0077 & 0.0091 & 0.0069 \\
\hline 1 & 3 & 0.009 & 0.0017 & 0.0024 & 0.0019 & 0.0023 & 0.0018 & 0.002 & 0.002 & 0.0018 \\
\hline & 3 & 0.0116 & 0.0041 & 0.0048 & 0.0044 & 0.0047 & 0.0044 & 0.0043 & 0.0046 & 0.0038 \\
\hline & & & 0.0094 & 0.0102 & 0.0099 & 0.0096 & 0.0102 & 0.0089 & 0.011 & 0.0083 \\
\hline-1 & 3 & 0.0084 & 0.0016 & 0.0023 & 0.0017 & 0.0021 & 0.0019 & 0.0021 & 0.0023 & 0.002 \\
\hline & 3 & 0.0115 & 0.0039 & 0.0047 & 0.0042 & 0.0046 & 0.0046 & 0.0044 & 0.0055 & 0.0045 \\
\hline & & & 0.0087 & 0.0097 & 0.0096 & 0.0094 & 0.0101 & 0.0089 & 0.0123 & 0.0097 \\
\hline-3 & 25 & 0.0087 & 0.0015 & & 0.0012 & 0.0014 & 0.0006 & 0.0007 & $5 \mathrm{E}-05$ & $5 \mathrm{E}-05$ \\
\hline & 3 & 0.0123 & 0.0039 & 0.0049 & 0.0026 & 0.0029 & 0.0019 & 0.0018 & 0.0001 & 0.0001 \\
\hline & & & 0.009 & 0.0097 & 0.0065 & 0.0063 & 0.0048 & 0.0041 & 0.0004 & 0.0003 \\
\hline 3 & 25 & 0.0085 & 0.0019 & 0.0026 & 0.0009 & 0.0011 & 0.0007 & 0.0008 & $6 \mathrm{E}-05$ & $5 \mathrm{E}-05$ \\
\hline & 3 & 0.0121 & 0.0041 & 0.0049 & 0.0025 & 0.0025 & 0.0018 & 0.0017 & 0.0002 & 0.0002 \\
\hline & & & 0.0088 & 0.0095 & 0.0056 & 0.0054 & 0.0045 & 0.004 & 0.0003 & 0.0002 \\
\hline 3 & 25 & 0.0111 & 0.0028 & 0.0038 & 0.0009 & 0.0012 & 0.0008 & 0.0009 & 0.0003 & 0.0002 \\
\hline \multirow[t]{2}{*}{1} & 7 & 0.0114 & 0.0058 & 0.0068 & 0.0023 & 0.0025 & 0.0022 & 0.0021 & 0.0009 & 0.0006 \\
\hline & & & 0.0116 & 0.0125 & 0.0059 & 0.0057 & 0.0057 & 0.005 & 0.0024 & 0.0018 \\
\hline-3 & 25 & 0.0109 & 0.0029 & & 0.0011 & 0.0014 & 0.0008 & 0.0009 & 0.0003 & 0.0003 \\
\hline & 7 & 0.0114 & 0.0058 & 0.0068 & 0.0029 & 0.0031 & 0.0022 & 0.0021 & 0.0009 & 0.0007 \\
\hline & & & 0.0112 & 0.012 & 0.0063 & 0.0061 & 0.0055 & 0.0048 & 0.0024 & 0.0017 \\
\hline-1 & 3 & 0.0096 & 0.002 & 0.0029 & 0.0017 & 0.002 & 0.0016 & 0.0018 & 0.0015 & 0.0013 \\
\hline & 7 & 0.0119 & 0.0047 & 0.0055 & 0.0039 & 0.0042 & 0.0042 & 0.004 & 0.0036 & 0.0029 \\
\hline & & & 0.0099 & 0.0107 & 0.0086 & 0.0084 & 0.0091 & 0.008 & 0.0077 & 0.006 \\
\hline 1 & 3 & 0.0094 & 0.0019 & 0.0027 & 0.0017 & 0.0021 & 0.0016 & 0.0017 & 0.0011 & 0.001 \\
\hline & 7 & 0.0117 & 0.0043 & 0.0052 & 0.0038 & 0.0041 & 0.0039 & 0.0037 & 0.0026 & 0.0021 \\
\hline & & & 0.0097 & 0.0105 & 0.009 & 0.0088 & 0.0089 & 0.0078 & 0.007 & 0.0054 \\
\hline
\end{tabular}


BEVERSDORF \& SA

Table 3: Type I Error Rates for Right-Tailed Test, 0.05 Level of Significance

\begin{tabular}{|c|c|c|c|c|c|c|c|c|c|c|}
\hline \multirow[b]{2}{*}{ Skewness } & \multirow[b]{2}{*}{ Kurtosis } & \multicolumn{3}{|c|}{$\mathrm{RHO}=0$} & \multicolumn{2}{|c|}{$\mathrm{RHO}=0.5$} & \multicolumn{2}{|c|}{$\mathrm{RHO}=0.7$} & \multicolumn{2}{|c|}{$\mathrm{RHO}=0.9$} \\
\hline & & $\begin{array}{c}\text { Pearson } \\
\text { Spearman }\end{array}$ & $\mathrm{r}_{\mathrm{L}}$ & $\mathrm{r}_{\mathrm{F}}$ & $\mathrm{r}_{\mathrm{L}}$ & $\mathrm{r}_{\mathrm{F}}$ & $\mathrm{r}_{\mathrm{L}}$ & $r_{F}$ & $\mathrm{r}_{\mathrm{L}}$ & $r_{F}$ \\
\hline 3 & 25 & 0.0635 & 0.0479 & 0.0474 & 0.1168 & 0.1171 & 0.1419 & 0.142 & 0.1671 & 0.1666 \\
\hline \multirow[t]{2}{*}{3} & 25 & 0.0511 & 0.0555 & 0.0544 & 0.1308 & 0.1303 & 0.1579 & 0.1571 & 0.1835 & 0.1822 \\
\hline & & & 0.0642 & 0.0626 & 0.1458 & 0.1447 & 0.1746 & 0.1733 & 0.2006 & 0.1985 \\
\hline-3 & 25 & 0.0654 & 0.05 & 0.0494 & 0.1179 & 0.1181 & 0.1431 & 0.1432 & 0.1664 & 0.1661 \\
\hline \multirow[t]{2}{*}{-3} & 25 & 0.0524 & 0.0573 & 0.0564 & 0.132 & 0.1316 & 0.1578 & 0.1571 & 0.1826 & 0.1816 \\
\hline & & & 0.0662 & 0.0645 & 0.1465 & 0.1454 & 0.1742 & 0.1729 & 0.1998 & 0.198 \\
\hline-1 & 7 & 0.0528 & 0.0362 & 0.0357 & 0.0508 & 0.051 & 0.0587 & 0.0588 & 0.0674 & 0.0672 \\
\hline \multirow[t]{2}{*}{-1} & 7 & 0.0532 & 0.0441 & 0.0429 & 0.0616 & 0.0612 & 0.0699 & 0.0694 & 0.0799 & 0.0787 \\
\hline & & & 0.0538 & 0.0517 & 0.0737 & 0.0728 & 0.0828 & 0.0817 & 0.0936 & 0.092 \\
\hline 1 & 7 & 0.0533 & 0.0356 & 0.0348 & 0.0511 & 0.0514 & 0.0598 & 0.0599 & 0.0683 & 0.0681 \\
\hline & 7 & 0.0512 & 0.0442 & 0.043 & 0.0614 & 0.0611 & 0.0707 & 0.0702 & 0.0804 & 0.0795 \\
\hline & & & 0.0542 & 0.0523 & 0.0737 & 0.0728 & 0.0827 & 0.0816 & 0.0937 & 0.0922 \\
\hline 1 & 3 & 0.0539 & 0.0353 & 0.0347 & 0.0431 & 0.0433 & 0.0461 & 0.0462 & 0.0497 & 0.0495 \\
\hline & 3 & 0.0532 & 0.0442 & 0.0428 & 0.0525 & 0.0522 & 0.0566 & 0.0563 & 0.0601 & 0.0591 \\
\hline & & & 0.055 & 0.0528 & 0.064 & 0.0633 & 0.0685 & 0.0676 & 0.0725 & 0.0708 \\
\hline-1 & 3 & 0.0535 & 0.0357 & 0.035 & 0.0424 & 0.0424 & 0.0469 & 0.047 & 0.0495 & 0.0492 \\
\hline \multirow[t]{2}{*}{-1} & 3 & 0.0529 & 0.0443 & 0.0431 & 0.0525 & 0.0523 & 0.0569 & 0.0565 & 0.0604 & 0.0598 \\
\hline & & & 0.0544 & 0.0524 & 0.0635 & 0.0625 & 0.0694 & 0.0684 & 0.0728 & 0.0714 \\
\hline-3 & 25 & 0.0569 & 0.0394 & 0.0389 & 0.0666 & 0.0668 & 0.0774 & 0.0775 & 0.0943 & 0.0937 \\
\hline \multirow[t]{2}{*}{-1} & 3 & 0.0526 & 0.0475 & 0.0465 & 0.0783 & 0.078 & 0.0915 & 0.0909 & 0.1151 & 0.1136 \\
\hline & & & 0.0578 & 0.0559 & 0.0921 & 0.091 & 0.1078 & 0.1065 & 0.139 & 0.136 \\
\hline 3 & 25 & 0.0582 & 0.0401 & & & 0.0668 & 0.0796 & 0.0797 & 0.0968 & 0.0964 \\
\hline & 3 & 0.0524 & 0.0494 & 0.048 & 0.0794 & 0.079 & 0.0931 & 0.0926 & 0.1168 & 0.115 \\
\hline & & & 0.0591 & 0.0573 & 0.0937 & 0.0927 & 0.1096 & 0.1082 & 0.1412 & 0.1383 \\
\hline 3 & 25 & 0.0576 & 0.0404 & 0.0399 & 0.0781 & 0.0784 & 0.0925 & 0.0926 & 0.1068 & 0.1063 \\
\hline & 7 & 0.0533 & 0.0486 & 0.0474 & 0.0906 & 0.0902 & 0.1061 & 0.1055 & 0.124 & 0.1226 \\
\hline & & & 0.0585 & 0.0567 & 0.1044 & 0.1032 & 0.122 & 0.1207 & 0.1431 & 0.1412 \\
\hline-3 & 25 & 0.0585 & 0.0409 & 0.0403 & 0.0773 & 0.0776 & 0.0925 & 0.0926 & 0.1081 & 0.1078 \\
\hline \multirow[t]{2}{*}{-1} & 7 & 0.0532 & 0.0491 & 0.048 & 0.0897 & 0.0893 & 0.1065 & 0.1059 & 0.1256 & 0.1243 \\
\hline & & & 0.0591 & 0.0575 & 0.1042 & 0.103 & 0.1234 & 0.122 & 0.1444 & 0.1423 \\
\hline-1 & 3 & 0.0523 & 0.0344 & 0.0337 & 0.0464 & 0.0467 & 0.051 & 0.0511 & 0.0561 & 0.0558 \\
\hline \multirow[t]{2}{*}{-1} & 7 & 0.0523 & 0.043 & 0.0418 & 0.0565 & 0.0562 & 0.0618 & 0.0613 & 0.0677 & 0.0667 \\
\hline & & & 0.0533 & 0.051 & 0.0681 & 0.0674 & 0.0743 & 0.0731 & 0.0812 & 0.0797 \\
\hline 1 & 3 & 0.0521 & 0.0349 & 0.0345 & 0.0466 & 0.0467 & 0.0507 & 0.0507 & 0.0562 & 0.0558 \\
\hline & 7 & 0.0516 & 0.0431 & 0.0419 & 0.0571 & 0.0568 & 0.0607 & 0.0602 & 0.0669 & 0.0661 \\
\hline & & & 0.0529 & 0.051 & 0.0686 & 0.0677 & 0.0732 & 0.0721 & 0.0798 & 0.0781 \\
\hline
\end{tabular}




\section{TESTS FOR CORRELATION ON BIVARIATE NON-NORMAL DATA}

Table 4: Type I Error Rates for Two-Tailed Test, 0.05 Level of Significance

\begin{tabular}{|c|c|c|c|c|c|c|c|c|c|c|}
\hline \multirow[b]{2}{*}{ Skewness } & \multirow[b]{2}{*}{ Kurtosis } & \multicolumn{3}{|c|}{$\mathrm{RHO}=0$} & \multicolumn{2}{|c|}{$\mathrm{RHO}=0.5$} & \multicolumn{2}{|c|}{$\mathrm{RHO}=0.7$} & \multicolumn{2}{|c|}{$\mathrm{RHO}=0.9$} \\
\hline & & $\begin{array}{c}\text { Pearson } \\
\text { Spearman }\end{array}$ & $r_{L}$ & $r_{F}$ & $r_{L}$ & $r_{F}$ & $\mathrm{r}_{\mathrm{L}}$ & $\mathrm{r}_{\mathrm{F}}$ & $r_{L}$ & $r_{F}$ \\
\hline 3 & 25 & 0.0648 & 0.0376 & 0.0395 & 0.0757 & 0.0789 & 0.0974 & 0.0997 & 0.1223 & 0.1222 \\
\hline \multirow[t]{2}{*}{3} & 25 & 0.0532 & 0.0499 & 0.0508 & 0.0946 & 0.0961 & 0.122 & 0.1218 & 0.152 & 0.1484 \\
\hline & & & 0.0659 & 0.0658 & 0.1193 & 0.1184 & 0.1525 & 0.1486 & 0.1879 & 0.1793 \\
\hline-3 & 25 & 0.064 & 0.0368 & 0.0389 & 0.0773 & 0.0806 & 0.0998 & 0.1022 & 0.1235 & 0.1232 \\
\hline \multirow[t]{2}{*}{-3} & 25 & 0.0535 & 0.0494 & 0.0504 & 0.0976 & 0.0989 & 0.1251 & 0.1249 & 0.1523 & 0.1481 \\
\hline & & & 0.0652 & 0.065 & 0.1224 & 0.1217 & 0.1561 & 0.1525 & 0.1863 & 0.1782 \\
\hline-1 & 7 & 0.0543 & 0.0253 & 0.0274 & 0.0306 & 0.032 & 0.0352 & 0.036 & 0.0425 & 0.0416 \\
\hline \multirow[t]{2}{*}{-1} & 7 & 0.0539 & 0.0378 & 0.0389 & 0.045 & 0.0452 & 0.051 & 0.0506 & 0.0592 & 0.0564 \\
\hline & & & 0.0554 & 0.0554 & 0.0646 & 0.0629 & 0.0729 & 0.0701 & 0.0823 & 0.0766 \\
\hline 1 & 7 & 0.0534 & 0.0254 & 0.0272 & 0.0314 & 0.033 & 0.0369 & 0.0377 & 0.0418 & 0.0414 \\
\hline & 7 & 0.0544 & 0.0376 & 0.0387 & 0.045 & 0.0457 & 0.0526 & 0.0519 & 0.0599 & 0.0569 \\
\hline & & & 0.0545 & 0.0544 & 0.0651 & 0.0638 & 0.0741 & 0.071 & 0.0822 & 0.0763 \\
\hline 1 & 3 & 0.0513 & 0.0233 & 0.025 & 0.0268 & 0.0281 & 0.0301 & 0.03 & 0.0324 & 0.031 \\
\hline & 3 & 0.054 & 0.0353 & 0.0363 & 0.0407 & 0.0406 & 0.0443 & 0.0429 & 0.0477 & 0.0443 \\
\hline & & & 0.0526 & 0.0524 & 0.0601 & 0.0582 & 0.0637 & 0.0604 & 0.0691 & 0.0626 \\
\hline-1 & 3 & 0.0524 & 0.0238 & 0.0256 & 0.0273 & 0.0285 & 0.029 & 0.0291 & 0.032 & 0.031 \\
\hline \multirow[t]{2}{*}{-1} & 3 & 0.0555 & 0.0361 & 0.037 & 0.0401 & 0.0401 & 0.0436 & 0.0422 & 0.0472 & 0.0439 \\
\hline & & & 0.0537 & 0.0536 & 0.06 & 0.058 & 0.0643 & 0.0606 & 0.0687 & 0.0619 \\
\hline-3 & 25 & 0.0547 & 0.0265 & & 0.0379 & 0.04 & 0.0432 & 0.0448 & 0.037 & 0.0397 \\
\hline \multirow[t]{2}{*}{-1} & 3 & 0.0556 & 0.0388 & 0.0398 & 0.0535 & 0.0545 & 0.061 & 0.0613 & 0.0546 & 0.0565 \\
\hline & & & 0.0557 & 0.0556 & 0.0743 & 0.0734 & 0.0836 & 0.0819 & 0.0788 & 0.0788 \\
\hline 3 & 25 & 0.0541 & 0.0259 & 0.0279 & 0.0374 & 0.0395 & 0.0429 & 0.0446 & 0.0388 & 0.0415 \\
\hline \multirow[t]{2}{*}{1} & 3 & 0.0552 & 0.0384 & 0.0393 & 0.0533 & 0.0539 & 0.0608 & 0.0606 & 0.0571 & 0.0584 \\
\hline & & & 0.0553 & 0.0551 & 0.074 & 0.0734 & 0.0837 & 0.0821 & 0.0805 & 0.0805 \\
\hline 3 & 25 & 0.0571 & 0.0293 & 0.0311 & 0.0451 & 0.0473 & 0.055 & 0.057 & 0.0559 & 0.0579 \\
\hline & 7 & 0.0545 & 0.0415 & 0.0426 & 0.0612 & 0.0622 & 0.0739 & 0.0742 & 0.0752 & 0.0754 \\
\hline & & & 0.0581 & 0.058 & 0.0827 & 0.0818 & 0.098 & 0.0958 & 0.1 & 0.0978 \\
\hline-3 & 25 & 0.0566 & 0.0302 & 0.0321 & 0.0448 & 0.0474 & 0.055 & 0.057 & 0.0551 & 0.0572 \\
\hline \multirow[t]{2}{*}{-1} & 7 & 0.0543 & 0.0425 & 0.0435 & 0.0618 & 0.0627 & 0.0742 & 0.0743 & 0.0747 & 0.0747 \\
\hline & & & 0.0578 & 0.0577 & 0.0833 & 0.0826 & 0.0978 & 0.0956 & 0.0989 & 0.0967 \\
\hline-1 & 3 & 0.0516 & 0.0235 & 0.0251 & 0.0275 & 0.0289 & 0.0298 & 0.0301 & 0.0317 & 0.0311 \\
\hline \multirow[t]{2}{*}{-1} & 7 & 0.0536 & 0.0353 & 0.0365 & 0.0413 & 0.0413 & 0.0438 & 0.043 & 0.0463 & 0.0444 \\
\hline & & & 0.0528 & 0.0527 & 0.0606 & 0.0589 & 0.0642 & 0.0612 & 0.0676 & 0.0626 \\
\hline 1 & 3 & 0.0518 & 0.0234 & 0.0254 & 0.0282 & 0.0293 & 0.031 & 0.0317 & 0.0316 & 0.0311 \\
\hline & 7 & 0.0556 & 0.0358 & 0.037 & 0.042 & 0.0422 & 0.0454 & 0.0444 & 0.0465 & 0.0443 \\
\hline & & & 0.0529 & 0.0529 & 0.0612 & 0.0597 & 0.0663 & 0.0629 & 0.0674 & 0.0621 \\
\hline
\end{tabular}


BEVERSDORF \& SA

Table 5: Power Results for Left-Tail Test when $\rho=0.7,0.05$ Level of Significance

\begin{tabular}{|c|c|c|c|c|c|c|c|c|c|}
\hline \multirow[b]{2}{*}{ Skewness } & \multirow[b]{2}{*}{ Kurtosis } & \multicolumn{2}{|c|}{$\mathrm{RHO}=0.7$} & \multicolumn{2}{|c|}{$\mathrm{RHO}=0.5$} & \multicolumn{2}{|c|}{$\mathrm{RHO}=0.3$} & \multicolumn{2}{|c|}{$\mathrm{RHO}=0.1$} \\
\hline & & $r_{L}$ & $\mathrm{r}_{\mathrm{F}}$ & $r_{L}$ & $\mathrm{r}_{\mathrm{F}}$ & $r_{L}$ & $\mathrm{r}_{\mathrm{F}}$ & $r_{L}$ & $r_{F}$ \\
\hline 3 & 25 & 0.0323 & 0.0263 & 0.1658 & 0.1442 & 0.3906 & 0.3583 & 0.648 & 0.6175 \\
\hline \multirow[t]{2}{*}{3} & 25 & 0.0421 & 0.0344 & 0.1964 & 0.1734 & 0.4354 & 0.4012 & 0.6858 & 0.658 \\
\hline & & 0.0537 & 0.0444 & 0.2300 & 0.2033 & 0.4781 & 0.4445 & 0.7195 & 0.6934 \\
\hline-3 & 25 & 0.0325 & 0.0261 & 0.1633 & 0.1429 & 0.3891 & 0.3565 & 0.6489 & 0.6194 \\
\hline \multirow[t]{2}{*}{-3} & 25 & 0.0429 & 0.0348 & 0.1948 & 0.1705 & 0.4338 & 0.4000 & 0.6875 & 0.6583 \\
\hline & & 0.0549 & 0.0451 & 0.2283 & 0.2021 & 0.4756 & 0.4420 & 0.7212 & 0.6951 \\
\hline-1 & 7 & 0.0289 & 0.0241 & 0.1612 & 0.1424 & 0.3909 & 0.3577 & 0.639 & 0.6059 \\
\hline \multirow[t]{2}{*}{-1} & 7 & 0.0369 & 0.0307 & 0.1919 & 0.1685 & 0.4374 & 0.4023 & 0.6809 & 0.6495 \\
\hline & & 0.0469 & 0.0387 & 0.2257 & 0.1986 & 0.4824 & 0.4466 & 0.7195 & 0.6891 \\
\hline 1 & 7 & 0.0292 & 0.0245 & 0.161 & 0.1409 & 0.391 & 0.3587 & 0.6357 & 0.6046 \\
\hline \multirow[t]{2}{*}{1} & 7 & 0.0374 & 0.0312 & 0.1917 & 0.1682 & 0.4366 & 0.4021 & 0.6784 & 0.6460 \\
\hline & & 0.0475 & 0.0392 & 0.2245 & 0.1983 & 0.4821 & 0.4460 & 0.7179 & 0.6865 \\
\hline 1 & 3 & 0.0321 & 0.0268 & 0.1696 & 0.1494 & 0.3985 & 0.3669 & 0.6369 & 0.6059 \\
\hline & 3 & 0.0411 & 0.034 & 0.1998 & 0.1767 & 0.443 & 0.4086 & 0.6776 & 0.6469 \\
\hline & & 0.0527 & 0.0434 & 0.2335 & 0.2069 & 0.488 & 0.452 & 0.7166 & 0.6859 \\
\hline-1 & 3 & 0.0326 & 0.0271 & 0.1706 & 0.1501 & 0.3986 & 0.3667 & 0.6393 & 0.6078 \\
\hline \multirow[t]{2}{*}{-1} & 3 & 0.0411 & 0.0346 & 0.2013 & 0.1777 & 0.4428 & 0.4089 & 0.6803 & 0.6492 \\
\hline & & 0.0517 & 0.0433 & 0.2344 & 0.2081 & 0.4881 & 0.4522 & 0.7184 & 0.6879 \\
\hline-3 & 25 & 0.0189 & 0.0153 & 0.1476 & 0.1285 & 0.3818 & 0.3503 & 0.6333 & 0.6034 \\
\hline \multirow[t]{2}{*}{-1} & 3 & 0.0250 & 0.0203 & 0.1759 & 0.1542 & 0.4253 & 0.3925 & 0.6744 & 0.6434 \\
\hline & & 0.0328 & 0.0266 & 0.2066 & 0.1819 & 0.4689 & 0.4346 & 0.7112 & 0.6828 \\
\hline 3 & 25 & 0.0199 & 0.0163 & 0.1461 & 0.1270 & 0.3800 & 0.349 & 0.6366 & 0.6065 \\
\hline & 3 & 0.0260 & 0.0212 & 0.1746 & 0.1522 & 0.4239 & 0.3905 & 0.6778 & 0.6468 \\
\hline & & 0.0341 & 0.0274 & 0.206 & 0.181 & 0.4671 & 0.4327 & 0.7147 & 0.6859 \\
\hline 3 & 25 & 0.0217 & 0.0175 & 0.1471 & 0.1275 & 0.3773 & 0.3457 & 0.6382 & 0.6062 \\
\hline & 7 & 0.0288 & 0.023 & 0.1768 & 0.154 & 0.4222 & 0.388 & 0.6793 & 0.6481 \\
\hline & & 0.0377 & 0.0306 & 0.2087 & 0.183 & 0.4672 & 0.4316 & 0.7169 & 0.6879 \\
\hline-3 & 25 & 0.0219 & 0.0178 & 0.1479 & 0.1286 & 0.3798 & 0.3478 & 0.6397 & 0.6078 \\
\hline \multirow[t]{2}{*}{-1} & 7 & 0.0288 & 0.0235 & 0.1778 & 0.155 & 0.4243 & 0.3905 & 0.6795 & 0.6497 \\
\hline & & 0.0375 & 0.0303 & 0.2094 & 0.1843 & 0.4694 & 0.4341 & 0.7171 & 0.6876 \\
\hline-1 & 3 & 0.0296 & 0.0244 & 0.1642 & 0.1444 & 0.3955 & 0.363 & 0.6361 & 0.6045 \\
\hline \multirow[t]{2}{*}{-1} & 7 & 0.0373 & 0.0313 & 0.1942 & 0.1711 & 0.4399 & 0.4062 & 0.6771 & 0.6463 \\
\hline & & 0.0469 & 0.0392 & 0.2268 & 0.2008 & 0.4851 & 0.4491 & 0.7167 & 0.6849 \\
\hline 1 & 3 & 0.0298 & 0.025 & 0.1659 & 0.1458 & 0.3942 & 0.3621 & 0.6356 & 0.6032 \\
\hline \multirow[t]{2}{*}{1} & 7 & 0.0379 & 0.0315 & 0.1964 & 0.1731 & 0.4385 & 0.4053 & 0.6768 & 0.6458 \\
\hline & & 0.0475 & 0.0396 & 0.2289 & 0.2031 & 0.4835 & 0.4482 & 0.7159 & 0.6856 \\
\hline
\end{tabular}

